\title{
Influencia del campo magnético estático en la turbidez de la cerveza de alta gravedad
}

Influence of application static magnetic on the turbidity of high gravity beer

\section{Autores | Authors}

Matilde Anaya VILLALPANDA Hilda Cobo ALMAGUER

Instituto de Investigaciones para la Industria Alimentaria (IIIA)

Departamento de Microbiología de Vicedirección de Ciencia

Playa/La Habana - Cuba e-mail:mavillal@iiia.edu.cu hildacobo@iiia.edu.cu

\section{Carlos Manuel Acea FIALLO}

Grupo ECOSOL

Playa/La Habana - Cuba e-mail:dingenieria@geca.minaz.cu

\section{凶 João Batista de ALMEIDA E}

SILVA

Universidade de São Paulo (USP) Escola de Engenharia de Lorena (EEL) Departamento de Biotecnologia Rod. Itajuba-Lorena, KM 74,5 CEP: $12602-810$ Lorena/SP - Brasil e-mail: joaobatista@debiq.eel.usp.br

\ Autor Correspondente / Corresponding Author

Recebido / Received: 25/09/2012 Aprovado / Approved: 14/11/2013 Publicado / Published: dez./2013

\section{Resumen}

Se aplicó el campo magnético estático a la cerveza de alta gravedad en tres etapas distintas del proceso, a partir de un diseño multifactorial D-óptimo de tres factores (intensidad de corriente, tiempo de residencia y tipo de cerveza). Se determinó la turbidez variable de respuesta por la diferencia de absorbancia por espectrofotometría a $580 \mathrm{~nm}$. Se obtuvo un modelo lineal en los rangos evaluados que relaciona dicha variable con el régimen de tratamiento magnético con efecto significativo sobre la aglutinación de las partículas coloidales en suspensión lo cual favorece la clarificación. La mejor combinación de factores estuvo en el rango de 0,40-0,55 A (100-120 mT) y tiempo de residencia 10 s en la cerveza no diluida y en la diluida, sin afectación de las características sensoriales.

Palabras-clave: Campo magnético estático; Cerveza de alta gravedad; Propiedades físico-químicas y sensoriales.

\section{Summary}

Static magnetic field was applied to high gravity beer in three different stages of the process, from a multivariate D-optimal design for three factors (current, residence time and type of beer). The response variable turbidity, was determined by difference of absorbance at $580 \mathrm{~nm}$. Linear model was obtained in the ranges tested relating the absorbance to the pattern of magnetic treatment, with significant effect on the binding of colloidal particles in suspension which favors clarification. The best combination of factors ranged from 0.40 to $0.55 \mathrm{~A}$ (100-120 mT) and time $10 \mathrm{~s}$ in non-diluted and diluted beer, without affecting the physical-chemical and sensory properties.

Key words: Static magnetic field; High-gravity beer; Physico-chemical and sensory properties. 


\section{Introducción}

La turbidez constituye un defecto de calidad de la cerveza cuando es filtrada y puede deberse a la presencia de partículas en suspensión como sustancias coloidales. Por tanto, la economía de la filtración depende en gran medida de tener todo el material no soluble sedimentado. De esta forma se pueden resolver aspectos de calidad y reducir los costos, así como mejorar la estabilidad de la cerveza y aumentar la retención de la espuma (ALMEIDA e SILVA, 2005).

En tal sentido el campo eléctrico pulsante es uno de los métodos más utilizados para la clarificación (McDONALD, 2000; YEOM, 2000). Debido a la interrelación entre corriente eléctrica y campo magnético inducido por ésta, se deduce la aplicación del campo electromagnético para la clarificación, con el empleo del tratamiento magnético (TM) en la clarificación de mieles, jugos de frutas, refinación de azúcar crudo y otros líquidos (ACEA, 2001; 2009; HERLEM, 2000). Esto indica la posibilidad del TM como complemento tecnológico del proceso cervecero.

Para las investigaciones, se obtiene el campo electromagnético a partir de una corriente eléctrica que pasa a través de una bobina tipo selenoide, por lo que será un campo magnético oscilante (CMO) dependiente de la frecuencia de la corriente eléctrica que lo induce. Si dicha corriente se rectifica a través de un banco de capacitores, se obtiene un campo magnético estático (CME), que podrá obtenerse luego por imanes permanentes de densidad de campo magnético equivalente al del estudio. Esta equivalencia constituye una ventaja tecnológica para el escalado de los resultados, en sistemas de flujo lento o moderado (ZAPATA et al., 2002).

Por este motivo, se evaluó el efecto del CME para disminuir la turbidez de la cerveza, evaluando diferentes regímenes de trabajo para el TM y sus afectaciones sobre las características físico-químicas y sensoriales del producto tratado.

\section{Materiale y Métodos}

El sistema hidráulico con acondicionador magnético acoplado a la tubería fue el mismo utilizado para determinar la influencia del CME en la formación de flóculos en una solución de azúcar crudo (ACEA, 2001; 2009), con similitud a los reportados en la literatura para su uso en cerveza (IVANOV et al., 1990; MIDDLETON, 2010; TIBOR, 2008; VASILENKO, 2003). El acondicionador magnético consta de dos pares de electroimanes tipo selenoide con núcleo de hierro, común dos a dos y polaridad sur-sur, formando dos zapatas polares que constituyen la región de tratamiento. Se alimentó con corriente alterna $(220 \mathrm{~V}, 1 \mathrm{~F}, 60 \mathrm{~Hz})$ através de un banco de capacitores para lograr CME por ajuste de intensidad de corriente con un amperímetro de escala 0,1-2,5 A, de precisión 0,1 A y densidad de campo de 5-200 mT. La tubería es de acero galvanizado de $13 \mathrm{~mm}$ de diámetro interno y $30 \mathrm{~cm}$ de longitud.

Para el presente estudio se empleó la cerveza obtenida de la fermentación de mosto de alta gravedad. Las muestras se tomaron de tres etapas distintas del proceso: no diluida a $17^{\circ} \mathrm{P}$ directo del contenedor en que se transporta desde diferentes cervecerías hacia la fábrica donde se envasa, diluida a $10^{\circ} \mathrm{P}$ que se envasa sin pasteurizar en toneles de acero inoxidable y recuperada de los tanques (devolución de toda la línea de envasado al final del turno de trabajo) con destino a la venta a granel a $8{ }^{\circ} \mathrm{P}$ (ANAYA et al., 2008a; b).

Se realizó un diseño experimental con el programa estadístico Design Expert ver. 6.0.1.0 (Stat-Ease, Minneapolis) con 20 corridas experimentales en un análisis multifactorial D-óptimo de tres factores, los cuales se realizaron de forma aleatoria (Tabla 1). Las variables de respuesta por evaluar fueron las propiedades físicoquímicas y la evaluación sensorial. Se aplicó el diseño a 5 muestras de cerveza terminada de cada una de las etapas seleccionadas (Tabla 2).

Los parámetros físico-químicos de las muestras se realizaron con el equipo Fermento-Start (Funke Gerber, Alemania): densidad $\left(\mathrm{g} / \mathrm{cm}^{3}\right)$, extracto real (\% $\mathrm{m} / \mathrm{m})$, extracto original $(\% \mathrm{~m} / \mathrm{m})$, grado alcohólico (\% $\mathrm{v} / \mathrm{v}$ ) y color (EBC). La turbidez se monitoreó sin filtrar la muestra, por diferencia de absorbancia a $580 \mathrm{~nm}$ medida con un espectrofotómetro (Jenway 6405 UV/ visible) y el pH mediante técnica potenciométrica (Meter mod. PHM 61, Dinamarca). El análisis sensorial se realizó con una comisión de cinco catadores de acuerdo con los cuales se multiplican por sus factores correspondientes según su importancia, expresando el valor en puntos experimentados en cerveza según lo establecido en el PAES para esta bebida (CUBA, 2001), que evaluaron los atributos espuma, fase líquida, olor, sabor y sensación bucal.

Tabla 1. Niveles de los factores del diseño experimental.

\begin{tabular}{cccc} 
Niveles & $\begin{array}{c}\text { Tiempo de } \\
\text { residencia (s) }\end{array}$ & $\begin{array}{c}\text { Factores } \\
\text { Intensidad de } \\
\text { corriente (A) }\end{array}$ & $\begin{array}{r}\text { Tipo de } \\
\text { cerveza }\end{array}$ \\
\hline 1 & 10 & $\begin{array}{c}0,60 \\
\text { (nivel alto) } \\
0,40\end{array}$ & No diluida \\
2 & 8 & Diluida \\
3 & - & - & Recuperada
\end{tabular}


Influencia del campo magnético estático en la turbidez de la cerveza de alta gravedad

VILLALPANDA, M. A. et al.

\section{Resultados y Discusión}

El procesamiento estadístico directo de las diferentes variables de respuesta en las variantes del diseño, no arrojó ningún modelo predictivo que explicara su comportamiento. Sin embargo, los resultados de las diferencias con respecto al control, sí se ajustaron a ecuaciones matemáticas.

El análisis de varianza del modelo obtenido para la diferencia de absorbancia demostró que el valor de $F(101,56)$ fue mayor que el de la tabla, por lo tanto se rechazó la hipótesis nula y se concluyó que el modelo y las diferencias fueron significativas ( $p \leq 0,05)$. La probabilidad de que el modelo lineal $\left(R^{2}=0,9667\right)$ no explique satisfactoriamente el comportamiento de las variables fue muy baja, por lo cual resultó adecuado. La Tabla 3 muestra los resultados de la diferencia respecto al control a partir de los cuales se obtuvo la siguiente ecuación:

$$
\mathrm{T}=\mathrm{X}_{0}-\mathrm{X}_{1} \cdot \mathrm{A}+\mathrm{X}_{2} \cdot \mathrm{B}-\mathrm{X}_{3} \cdot \mathrm{C}_{1}-\mathrm{X}_{4} \cdot \mathrm{C}_{2}
$$

donde $\mathrm{T}$ : turbidez; $\mathrm{X}_{0}$ : constante del modelo; $\mathrm{X}_{1}, \mathrm{X}_{2}, \mathrm{X}_{3} \mathrm{y}$ $X_{4}$ : coeficientes de regresión; $A$ : intensidad de corriente; B: flujo de cerveza; $C_{1}$ : cerveza no diluida; $C_{2}$ : cerveza diluida.

En la Tabla 3 se evidencia que se logró un incremento de la absorbancia (turbidez) respecto al control (dado por el signo negativo de las diferencias calculadas) en todo el rango de intensidad estudiado y en los tres tipos de cerveza. Es decir, que se obtuvo por este método físico un incremento de la desestabilización de las partículas en suspensión que podrán eliminarse posteriormente por filtración. En la Tabla 4 se aprecia que la variación de la diferencia de absorbancia fue significativa $(p \leq 0,05)$ en relación con la intensidad de corriente y el tempo de residencia, con un comportamiento lineal en los tres tipos de cerveza (Figura 1a-c).

Tabla 2. Valor promedio obtenido en la caracterización inicial de las muestras tratadas.

\begin{tabular}{lccc}
\multicolumn{1}{c}{ Propiedad } & No diluida & Diluida & Recuperada \\
\hline Absorbancia & $0,075(0,007)$ & $0,133(0,035)$ & $0,224(0,029)$ \\
pH & $4,4(0,1)$ & $4,2(0,1)$ & $3,7(0,3)$ \\
densidad $\left(\mathrm{g} / \mathrm{cm}^{3}\right)$ & $1,0076(0,02)$ & $1,0037(0,01)$ & $1,0024(0,04)$ \\
Extracto real $(\% \mathrm{~m} / \mathrm{m})$ & $2,46(0,59)$ & $1,70(0,80)$ & $1,63(0,40)$ \\
Extracto original $(\% \mathrm{~m} / \mathrm{m})$ & $16,87(0,43)$ & $10,40(0,31)$ & $8,2(0,15)$ \\
Grado alcohólico $(\% \mathrm{v} / \mathrm{v})$ & $7,97(0,06)$ & $5,10(0,02)$ & $3,47(0,51)$ \\
Color (EBC) & $9,03(0,04)$ & $8,75(0,01)$ & $8,22(0,04)$ \\
Sensorial (puntos) & $18,5(0,1)$ & $17,7(0,3)$ & $12,5(0,5)$ \\
\hline
\end{tabular}

Promedio de cinco muestras tratadas (desviación estándar).

Tabla 3. Matriz del diseño experimental.

\begin{tabular}{|c|c|c|c|c|c|}
\hline Corrida & Intensidad (A) & Tiempo (s) & Tipo cerveza & Absorbancia & pH \\
\hline 1 & 0,6 & 8 & No diluida & $-0,044$ & 0 \\
\hline 2 & 0,4 & 8 & No diluida & $-0,057$ & 0 \\
\hline 3 & 0,4 & 8 & Diluida & $-0,053$ & 0 \\
\hline 4 & 0,4 & 10 & No diluida & $-0,067$ & 0,1 \\
\hline 5 & 0,4 & 10 & Recuperada & $-0,05$ & 0,1 \\
\hline 6 & 0,5 & 10 & Diluida & $-0,059$ & 0 \\
\hline 7 & 0,45 & 9 & No diluida & $-0,056$ & 0 \\
\hline 8 & 0,6 & 8 & Diluida & $-0,039$ & 0 \\
\hline 9 & 0,6 & 8 & Recuperada & $-0,006$ & 0,1 \\
\hline 10 & 0,6 & 10 & Recuperada & $-0,023$ & 0,1 \\
\hline 11 & 0,6 & 10 & No diluida & $-0,059$ & 0 \\
\hline 12 & 0,5 & 9 & Recuperada & $-0,042$ & 0 \\
\hline 13 & 0,6 & 8 & No diluida & $-0,044$ & 0 \\
\hline 14 & 0,6 & 10 & Recuperada & $-0,023$ & 0,1 \\
\hline 15 & 0,4 & 8 & Recuperada & $-0,02$ & 0,1 \\
\hline 16 & 0,6 & 9 & Diluida & $-0,042$ & 0 \\
\hline 17 & 0,5 & 10 & Diluida & $-0,023$ & 0,1 \\
\hline 18 & 0,6 & 10 & No diluida & $-0,059$ & 0 \\
\hline 19 & 0,4 & 9 & Diluida & $-0,084$ & 0,1 \\
\hline 20 & 0,5 & 8 & Diluida & $-0,048$ & 0 \\
\hline
\end{tabular}




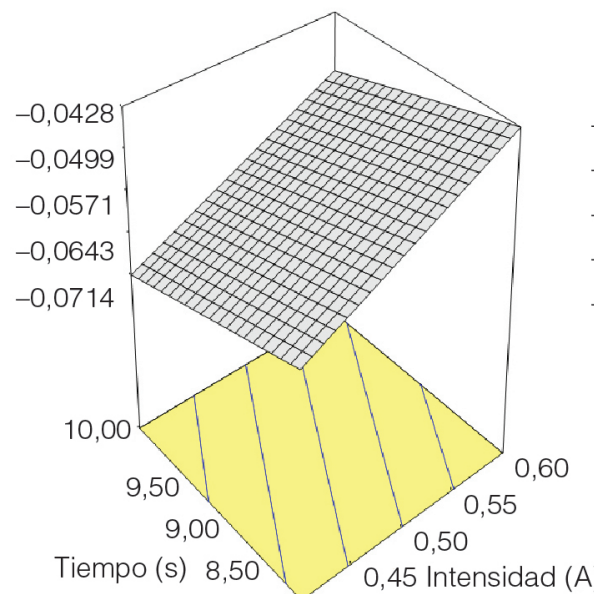
$8,00 \quad 0,40$

a)

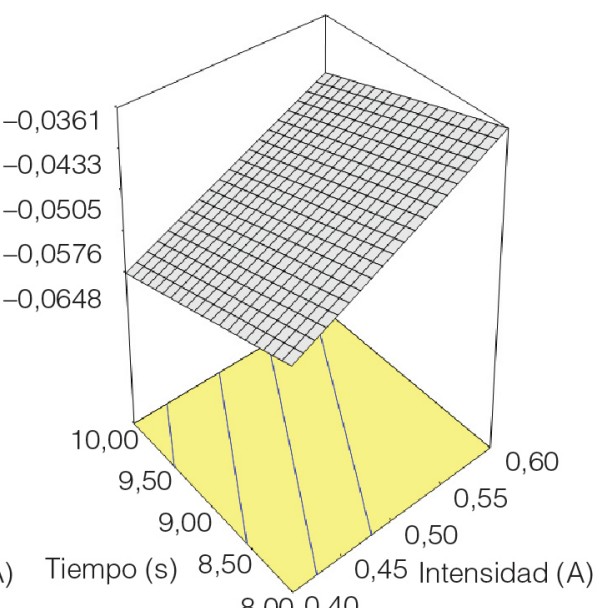

$8,000,40$

b)

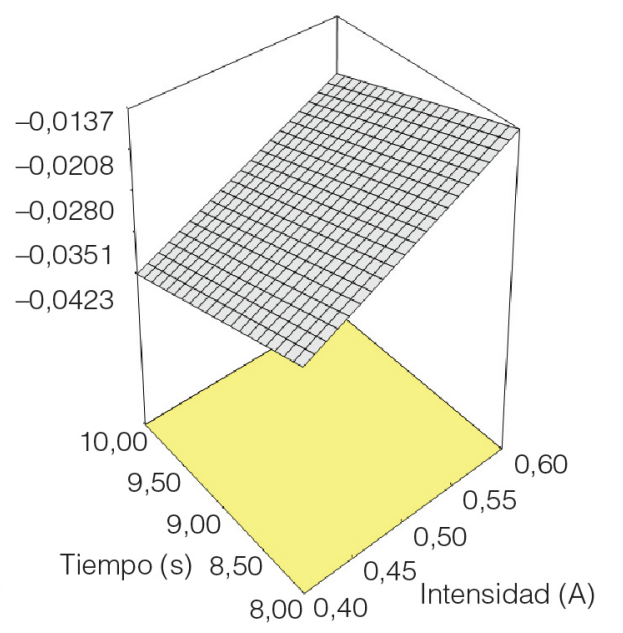

c)

Figura 1. Diferencia de absorbancia de las cervezas tratadas a $580 \mathrm{~nm}$ : a) no diluida; b) diluida; c) recuperada.

Tabla 4. Resultados del diseño de experimento a partir de las diferencias respecto al control.

\begin{tabular}{lccc}
\multicolumn{1}{c}{ Factor } & $\begin{array}{c}\text { Coeficientes } \\
\text { estimados }\end{array}$ & $\begin{array}{c}\text { Desviación } \\
\text { estándar }\end{array}$ & $\mathbf{p}$ \\
\hline Intercepto & $-0,045$ & $2,799 \mathrm{E}-003$ & 0,0021 \\
A (intensidad) & $9,105 \mathrm{E}-003$ & $3,220 \mathrm{E}-003$ & 0,0127 \\
B (tiempo de resid.) & $-5,224 \mathrm{E}-003$ & $3,123 \mathrm{E}-003$ & 0,1151 \\
C1 (concentrada) & $-0,012$ & $3,875 \mathrm{E}-003$ & 0,0021 \\
C2 (diluida) & $-5,282 \mathrm{E}-003$ & $3,912 \mathrm{E}-003$ & 0,0001 \\
\hline
\end{tabular}

El perfil para los tres tipos de cerveza (Figura 1a-c) fue muy similar, con disminución de la diferencia hasta valores cercanos a cero, lo cual se puede explicar por la formación máxima permisible de agregados coloidales o de flóculos en el sistema (ACEA, 2001). Además se aprecia que las máximas diferencias se obtuvieron en el rango de 0,40 a 0,50 A (equivalentes a 100 a 120 $\mathrm{mT}$ ) y que los mayores incrementos de absorbancia afectan a la cerveza no diluida y a la diluida (Figura 1a y b). Atendiendo a la composición de estas cervezas, este resultado puede indicar que el TM en el rango de intensidad de corriente estudiado actuó con mayor efectividad en el medio menos concentrado de partículas coloidales (menos turbio), como la cerveza no diluida y la diluida, que en el medio más concentrado en éstas (más turbio), como la cerveza recuperada (ha recibido el efecto de la variación de temperatura y el tiempo de permanencia en los tanques de recuperación). Este fenómeno se ha observado en numerosas investigaciones (ACEA, 2001; 2009) y se atribuye a la interferencia entre partículas coloidales y los coágulos ya formados, lo que dificulta la acción del TM en la floculación. En ese sentido, se ha visto mediante microfilmación que el CME atrae ordenadamente a estas partículas cargadas, formando flóculos cada vez más esféricos (ACEA, 2009). Los valores de inducción empleados en este sistema permiten la facilidad en el diseño de los imanes permanentes por colocar en los sistemas de tuberías con flujo moderado. El valor significativo del factor tiempo $(p \leq 0,05)$ evidencia que la mayor remoción se alcanzó a los $10 \mathrm{~s}$, ya que a mayor tiempo de residencia (menor flujo) mayor será el efecto del TM.

No fue posible encontrar un modelo con suficiente calidad $\left(R^{2}=0,5829\right)$ que explicara el comportamiento del $\mathrm{pH}$, lo cual coincide con lo informado por otros autores (VASILENKO, 2003). La variación de este parámetro es importante ya que puede influir en la coagulación y posterior precipitación de las proteínas (punto isoeléctrico) que forman los flóculos. La poca variación del mismo, la cual no fue significativa ( $p>0,05)$, permite corroborar la interpretación del efecto del CME sobre las partículas coloidales en suspensión. Estos resultados en las propiedades físico-químicas son similares a los obtenidos en otras investigaciones. Se plantea que, para variar significativamente estos parámetros, la energía aportada por el campo debe ser muy elevada (TIBOR, 2008), suficiente para la ruptura de enlaces por puente de hidrógeno, y provocar cambios sustanciales en la estructura de la sustancia (PANG e DENG, 2008).

En la evaluación sensorial no se obtuvieron diferencias significativas $(p>0,05)$ en los atributos analizados de las cervezas tratadas, con respecto a los controles. En particular, se pudo apreciar por todos los jueces una ligera disminución de la carbonatación debido a pequeñas pérdidas de $\mathrm{CO}_{2}$ que no pueden atribuirse al efecto del TM sino al traslado hacia el sistema utilizado (ZAPATA et al., 2002). La pérdida de $\mathrm{CO}_{2}$ incidió en la formación de la espuma, pero en ningún caso esta disminución invalidó la aceptación de la cerveza tratada, encontrándose una mejor retención de 
Influencia del campo magnético estático en la turbidez de la cerveza de alta gravedad

VILLALPANDA, M. A. et al.

la espuma creada respecto al control, consecuente con lo reportado en la literatura. Todos los tipos de cerveza mostraron un comportamiento similar evidenciando lo demostrado por diversas fuentes que el TM por lo general no afecta las características sensoriales de los productos (MIDDLETON, 2010; TIBOR, 2008; VASILENKO, 2003; ZAPATA et al., 2002).

\section{Conclusiones}

Se aplicó el campo magnético a la cerveza de alta gravedad en tres etapas diferentes del proceso y se determinó el régimen de tratamiento en 0,40 a 0,50 A (100 a $120 \mathrm{mT}$ ) y tiempo de residencia $10 \mathrm{~s}$ en cerveza no diluida y en diluida, sin afectaciones en las características sensoriales. Se obtuvo un modelo lineal que relaciona la absorbancia (turbidez) con el tratamiento aplicado, que mostró efecto significativo sobre la aglutinación de las partículas coloidales en suspensión. Esto es un aspecto positivo para la industria cervecera, ya que favorece el proceso de clarificación y, por lo tanto, las mismas pueden ser fácilmente removidas por la posterior filtración.

\section{Agradecimientos}

Los autores agradecen al Conselho Nacional de Desenvolvimento Científico e Tecnológico (CNPq), de Brasil, y al Ministerio de Educación Superior (MES) de Cuba.

\section{Referencias}

ACEA, C. Un solo golpe magnético. Revista ICIDCA, La Habana, v. 25, n. $2-3$, p. 24-29. 2001.

ACEA, C. Visualización del proceso de floculación magnética. In: CONFERENCIA INTERNACIONAL DE ELECTROMAGNETISMO APLICADO, 2009, Santiago de Cuba. Anais... Santiago de Cuba: CNEA, 2009.1 CD-ROM.

ALMEIDA E SILVA, J. B. Cerveja. In: VENTURINI FILHO, W. G. Tecnologia de Bebidas. São Paulo: Ed. Edgard Blucher, 2005. cap. 15, p. 347-382.

ANAYA, M.; ACEA, C.; GONZÁLEZ, B. In: Posibilidades del tratamiento magnético en la calidad de la cerveza. In: CONVENCIÓN INTERNACIONAL ALIMENTACIÓN SALUDABLE PARA LA COMUNIDAD Y EL TURISMO, 2008, La Habana. Anais... Habana: ACTAC, 2008a.
ANAYA, M.; ACEA, C.; GONZÁLEZ, B. Efecto del campo magnético en diferentes parámetros controlados en la industria cervecera. In: CONFERENCIA INTERNACIONAL SOBRE CIENCIA Y TECNOLOGÍA DE LOS ALIMENTOS, 2008, La Habana. Anais... Habana: ACTAC, 2008b. CD-ROM.

CUBA. Ministerio de la Industria Alimentaria. Manual de Instrucciones del Sistema de Control de la Calidad Procedimiento Analítico para el Control de la Calidad Sensorial de Cervezas. La Habana: Ministerio de la Industria Alimentaria, 2001. 18 p. Instrucción S.C.C 2.13.05.02-1. Capítulo II: Control de la Calidad.

HERLEM, B. J. Chlorine generation and disinfection by electroflotation. Journal Food Science, New York, v. 65, p. 834-837, 2000. http://dx.doi.org/10.1111/j.1365-2621.2000. tb13596.x

IVANOV, A. A.; SHTEPA, E. P.; GALIULIN, A. A.; KOLPAKCHI, A. P.; RUSAKOV, V. A.; ISAEVA, V. S.; PECHENINA, D. M. Stabilization of Beer. USSR-Patent: SU 1565 879, 1990.

McDONALD, C. J. Effects of pulsed electric fields on microorganisms in orange juice using electric field strengths of 30 and $50 \mathrm{kV} / \mathrm{cm}$. Jornal Food Science, New York, v. 65, p. 984-988, 2000. http://dx.doi.org/10.1111/j.1365-2621.2000. tb09404.x

MIDDLETON, D.L. Preventing Contaminant Build-up in Beer Lines. Patent US 5645697, 2010.

PANG, X.F.; DENG, B. Investigation of changes in properties of water under the action of a magnetic field. Science China Physics, Mechanics and Astronomy, Pequin, v. 51, n. 11, p. 1-12, 2008. http://dx.doi.org/10.1007/s11433-008-0182-7

TIBOR, N. Procedure to Manufacture and Use Beer and Other Types Liquids Treated with Neodimium Magnet. Patent W0102188, 2008

VASILENKO, V. Method and Apparatus for Preserving Perishable Products. Patent US 7682641, 2003.

YEOM, H. W. Effects of pulsed electric fields on activities of microorganisms and pectin methyl esterase in orange juice. Journal Food Science, New York, v. 65, p. 1359-1364, 2000. http://dx.doi.org/10.1111/j.1365-2621.2000.tb10612.x

ZAPATA, J. E.; MORENO, G.; MÁRQUEZ E. Efectos de los campos magnéticos sobre el crecimiento de Saccharomyces cerevisiae. Interciencia, Caracas, v. 27, n. 10, p. 544-550, 2002. 